\title{
Search for indications of fast rotation in the linear polarization of the magnetic white dwarf Grw $+70^{\circ} 8247^{\star}$
}

\author{
S. Friedrich ${ }^{1,2}$ and S. Jordan ${ }^{1}$ \\ 1 Institut für Theoretische Physik und Astrophysik, Universität Kiel, 24098 Kiel, Germany \\ 2 Astrophysikalisches Institut Potsdam, An der Sternwarte 16, 14482 Potsdam, Germany \\ Received 2 May 2000 / Accepted 5 December 2000
}

\begin{abstract}
Grw $+70^{\circ} 8247$ belongs to a small group of magnetic white dwarfs which despite repeated observations did not show any changes in their polarization over decades. The inferred rotation periods are of the order of hundred years which is not easy to understand if angular momentum is conserved during stellar evolution. An alternative possibility, which was to our knowledge never investigated, might be fast rotation with a period between some minutes and a couple of seconds. Assuming a mass of $1 M_{\odot}$ and a radius of $1 / 100 R_{\odot}$ the shortest possible period is about $10 \mathrm{~s}$. In order to test this hypothesis the linear polarization of Grw $+70^{\circ} 8247$ was measured at the $2.2 \mathrm{~m}$ telescope at the Calar Alto Observatory, Spain, with a time resolution of $3 \mathrm{~s}$ by trailing the linear polarized image of Grw $+70^{\circ} 8247$ over the CCD chip. The linear polarization was searched for periodicities in the range between $10 \mathrm{~s}$ and $4000 \mathrm{~s}$. Investigations with synthetic polarized data using the same window function and an assumed sinusoidal variation showed that variations with amplitudes of $40 \%$ relative to the amount of average linear polarization should be detectable. However, no periodicities at this or higher levels of significance were found. This would be in accordance with a recent report on slow rotation of GD 229 and G 240-72, but fast variations with lower amplitudes could not be ruled out.
\end{abstract}

Key words. stars: individual: Grw $+70^{\circ} 8247$ - stars: rotation - stars: white dwarfs

\section{Introduction}

Grw $+70^{\circ} 8247$ was detected in 1938 by Minkowski (1938) but it took until 1970 when it became clear through circular polarization measurements by Kemp et al. (1970) that it possesses a strong magnetic field. Despite repeated observations its polarization did not show any significant changes (West 1989). Slow rotation seems to be a common property of white dwarfs in general. Although mechanisms and time scales for angular momentum transport from cores to the envelope are not well understood, it became evident from initial-final mass relations derived for white dwarfs in open clusters (Weidemann \& Koester 1983; Koester \& Reimers 1996) that much of the initial angular momentum of a star could be lost. The mechanism most often discussed in this context is magnetic braking, i.e. the magnetically driven transfer of angular momentum of the star to a stellar wind and an expanding envelope during its evolution (e.g. Pacini 1970; Brecher \& Chanmugam 1978).

Send offprint requests to: S. Friedrich

* Based on observations collected at the German-Spanish Astronomical Center (DSAZ), Calar Alto, operated by the Max-Planck-Institut für Astronomie Heidelberg jointly with the Spanish National Commission for Astronomy.
The measurement of rotation velocities of white dwarfs is difficult. Due to the Stark effect and - in about $2-3 \%$ (Jordan 2000) of all white dwarfs - broadening by magnetic fields lines in the spectra have $F W H M$ of several $\AA$. The only practicable methods are the investigation of narrow NLTE line cores, period splitting of non-radial oscillation modes of variable white dwarfs (ZZ Ceti stars, e.g. Bergeron et al. 1993), and (spectro-)polarimetric observations of magnetic white dwarfs (e.g. Schmidt \& Norsworthy 1991).

A recent search for rotation by high resolution spectroscopy of the NLTE H $\alpha$ core in DA white dwarfs resulted in typical upper limits for $v \sin i$ of $15 \mathrm{~km} \mathrm{~s}^{-1}$ ( $i$ the angle between line of sight and rotation axis), corresponding to periods of hours or longer (Koester et al. 1998). Whereas most of the magnetic white dwarfs have rotation periods between some hours and days, RE J0317-853 has the shortest rotation period of merely $725 \mathrm{~s}$ corresponding to a rotational velocity of $\approx 50 \mathrm{~km} \mathrm{~s}^{-1}$ (Burleigh et al. 1999). GD 229 and G 240-72 on the other hand seem to show variability of their linear polarization on time scales of about ten years implying rotation periods of at least 100 years (Berdyugin \& Piirola 1999).

For magnetic white dwarfs there are three possible explanations why they apparently do not rotate. Firstly, they may have rotationally symmetric magnetic field 


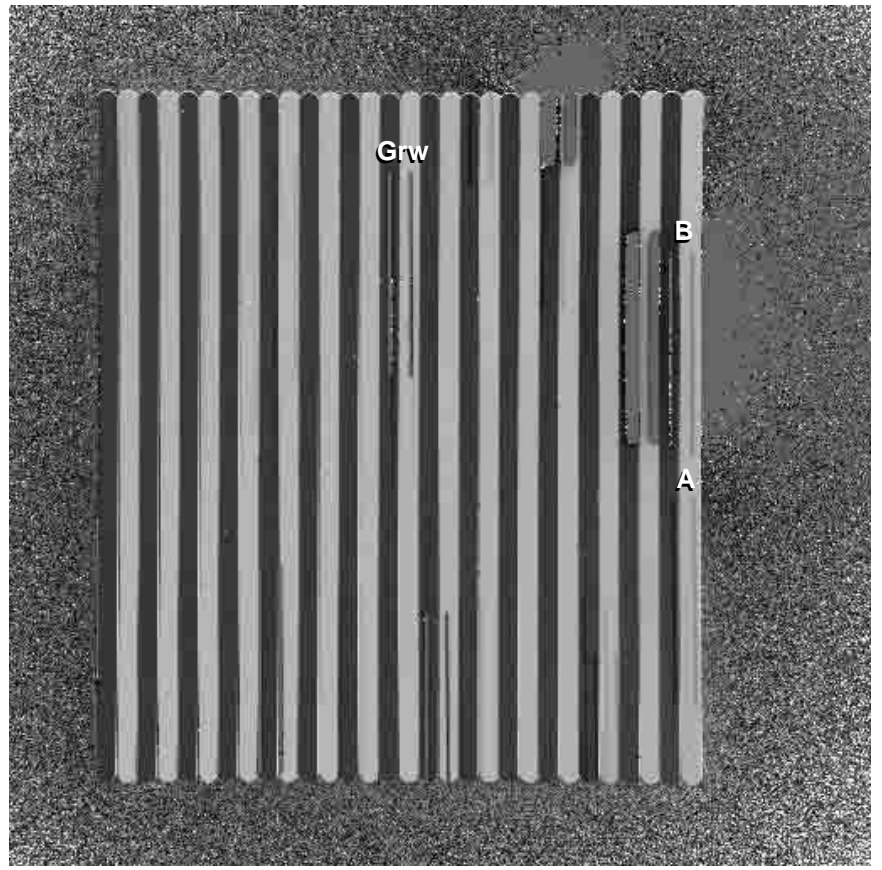

Fig. 1. One of the CCD images of the 1999 observing run. The trails of Grw $+70^{\circ} 8247$ and comparison stars are marked. The stripes of the mask are clearly visible

geometries. Secondly, they can have rotation periods greater than hundred years. Or thirdly, they may spin too rapidly $(P \leq 10 \mathrm{~min}$, Schmidt \& Norsworthy 1991) to be time-resolved as yet. It should be mentioned at this point that Angel et al. (1981) concluded from their data that rotation periods of less than $5 \mathrm{~min}$ for $\mathrm{Grw}+70^{\circ} 8247$ do not seem likely, but they did not discuss the basis of their statement in detail. Rotation periods as short as about $10 \mathrm{~s}$, the break-up limit when assuming a mass of $1 M_{\odot}$ and a radius of $1 / 100 R_{\odot}$, are theoretically possible. The goal of our observations was to test the third possibility, that means to look for rotation periods shorter than $10 \mathrm{~min}$ in $\mathrm{Grw}+70^{\circ} 8247$.

\section{Observation and data reduction}

Polarization is very sensitive to changes in the magnetic field geometry as seen by the observer which occurs for example when the magnetic field axis is tilted against the rotation axis. Then, rotation can be detected in periodic changes of the degree or angle of polarization.

For lack of a high-speed photometer with polarization optics at the Calar Alto Observatory, Spain, the $2.2 \mathrm{~m}$ telescope with the Calar Alto Faint Object Spectrograph (CAFOS, Meisenheimer 1998) for imaging polarimetry was proposed for the observation. The necessary time resolution is achieved by trailing the star images over the CCD. In order to test this observing strategy the program committee of the Calar Alto decided to let perform a test observation (half a night). This observation was carried out on August 3, 1999 by the Calar Alto staff. We performed a second observation on August 22 to August 25, 2000. Since this latter observation severely suffered from bad weather and some technical problems, our main conclusions are drawn from the test observation.

In addition to the rotatable halfwave plate and the Wollaston prism as beam splitter for measuring the linear polarization, a Johnson $B$ filter ( $F W H M 920 \AA)$ was used to narrow the wavelength range. Thus the cancelling of polarization over the wavelength range was reduced. For the polarization angle we have chosen 22 degrees (Landstreet \& Angel 1975) as a compromise of the values for the band pass of a $B$ filter cited in West (1989). However during the test observation the angle of the retarder plate was fixed to 22 degrees, which corresponds to a polarization angle of 44 degrees (Meisenheimer 2000) ${ }^{1}$; it remained fixed during the whole observation in order to minimize time gaps. We should emphasize that with a fixed retarder plate we cannot determine the polarization angle and the correct degree of linear polarization. Only the projection of the polarization vector parallel to this directional axis could be measured. Finally, a multi stripe mask with alternating blank and black stripes of $19^{\prime \prime}$ width each according to the beam separation of the Wollaston prism, was also inserted to fade out other stars. For the August 2000 observing run the same instrument, instrument configuration and observing strategy was chosen. In order to determine the polarization angle the angle of the retarder plate was not fixed but set to 19, 199, and 319 degrees for different exposures. In addition CCD polarimetry (without trailing) at six different retarder plate settings were performed.

In order to trail the star images over the CCD chip the telescope tracking speed was changed such that it results in an exposure time of $3 \mathrm{~s}$ per pixel parallel to the orientation of the stripe mask. This trailing velocity allows the detection of rotation periods as short as about $10 \mathrm{~s}$. Eight images, each with an exposure time of 1080 s, were obtained in this configuration in 1999. Due to a misunderstanding the trailing direction was south-west to northeast instead north-west to south-east. As a consequence an interference of the trail of $\mathrm{Grw}+70^{\circ} 8247$ with two nearby faint field stars occurred. If we assume that these stars are unpolarized this only leads to a somewhat lower polarization of Grw $+70^{\circ} 8247$. Since we are searching for variations the absolute value of the linear polarization is not important. In order to be able to identify aliases caused by the window function field stars marked with A and B in Fig. 1 were also extracted and their light curves searched for variations. During the 2000 observing run the trailing direction was north-west to south-east with no interference of neighbouring stars. Due to bad weather in two of the three nights only four exposures for each of the three retarder settings of 19, 199, and 319 degrees could

\footnotetext{
1 The zero point of the retarder is aligned to the north pole with an error of about \pm 1 degree and counted in the direction of increasing right ascension (Meisenheimer 2000). However the alignment could not be checked by observing a linear polarized standard star during the test observation.
} 
be obtained with exposure times variing between $643 \mathrm{~s}$ and $823 \mathrm{~s}$.

Data reduction of both observing runs was performed with standard IRAF routines for bias correction and flat fielding. The run of the trails was approximated by a cubic spline and their widths were determined by fitting Gaussian profiles. The extraction radius was set so that $99 \%(3 \sigma)$ of the intensity of the star was enclosed. The sky background for Grw $+70^{\circ} 8247$ was averaged over three columns immediately left and right to its trail. Comparison stars were treated in the same way. Due to the position of the comparison stars at the edge of the multi stripe mask (A) or near the bright star (B) in 1999 the sky background could be determined only on one side. The linear polarization was then determined as the normalized difference between the two adjacent trails. As a consequence of the inadequate sky subtraction the comparison stars show polarization of about $2 \%$ in 1999 . For comparison star B, however, the polarization drops to about zero when no interference with the trail of the bright star occurs anymore. Within the error margin the comparison star in 2000 is unpolarized. We are therefore convinced that our observing strategy is capable to detect whether a star is polarized or not.

Before linear polarized light curves could be constructed the starting pixel of each trail had to be defined. The trails rise well above the sky background in 1999 within a few pixels. According to a total exposure time of $1080 \mathrm{~s}$ and a velocity of $3 \mathrm{~s}$ per pixel each trail should have a length of 360 pixels. We therefore determined the centre between several points of the rise and the decrease of the trail. Then the starting pixel results from the mean of all centres by adding 180 pixels. The length of the trails could be reproduced with this method with a $1 \sigma$ uncertainty of 1.4 pixels for $\mathrm{Grw}+70^{\circ} 8247$. Starting pixels for the 2000 observation could be determined in a similar way with an error of 2 pixel. Nevertheless, we arbitrarily changed the starting pixel by up to 3 pixels to see how this would affect the results from the period search. No significant influence could be detected.

\section{Results}

The light curves of Grw $+70^{\circ} 8247$ show a mean linear polarization of about $1 \%$ with a scatter of $1 \%$ (rms) (Fig. 2) in 1999 which is lower than the published $3 \%$ for a Johnson $B$ filter (Fig. 1 in West 1989) due to the fact that the measurements were obtained for a polarization angle of 44 degrees. The calculation of the mean linear polarization and the mean polarization angle from the normalized flux differences of the 2000 trails $^{2}$ via Fourier transformation results in a polarization of $2.3 \% \pm 1 \%$ and a polarization angle of 19 degrees with an error of \pm 5 degrees and the uncertainty of the alignment of the retarder plate.

\footnotetext{
${ }^{2}$ Data reduction showed that the statistics of the polarimetry is too bad to get reliable results on polarization and polarization angle.
}

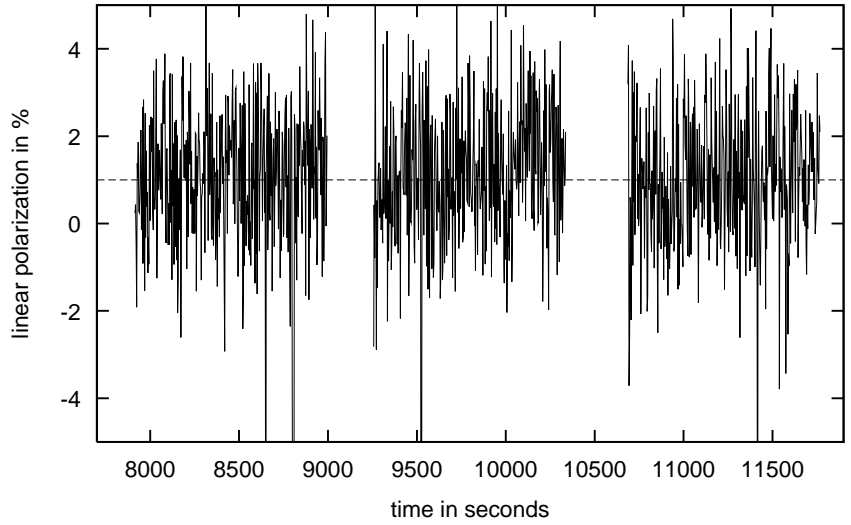

Fig. 2. Measured linear polarized light curves of Grw $+70^{\circ} 8247$ of three consecutive exposures for a fixed retarder angle of 22 degrees (see text). The dashed line marks the average linear polarization of $1 \%$ of the whole 1999 observing run

Unfortunately the observation of the linear polarized standard star HD 11831 (Bastien et al. 1988) was prevented by technical problems with CAFOS. However, if we assume that the alignment of the retarder plate is correct within an error of $\pm 1^{\circ}$ this would mean that the linear polarization and the polarization angle of $\mathrm{Grw}+70^{\circ} 8247 \mathrm{did}$ not change since the observations of West (1989).

Despite all these problems we searched for short term variations in the polarization by means of a $\chi^{2}$ procedure in the data of both observing runs. Since we can only measure the projection of the polarization on the directional axis in 1999 we are aware that the measured polarization is smaller than for the correct angle making it more difficult to discover variations. Trial periods were chosen according to the length of the corresponding observing intervals and range between $10 \mathrm{~s}$ and $4000 \mathrm{~s}$ for the 1999 and $10 \mathrm{~s}$ and up to $3000 \mathrm{~s}$ for the 2000 observation, respectively. In order to determine the $\chi^{2}$ value for each trial period the data were binned into 25 phase intervals. Then the $\chi^{2}$ value for the actual trial period is given by the normalized sum of the squares of the difference between each bin and the arithmetic mean of the linear polarization divided by the square of the error of the linear polarization. If no period is present in the observation the values of individual data points will scatter around the mean value and the $\chi^{2}$ will be about 1 . However, if variations are present they will systematically differ from the mean and therefore the $\chi^{2}$ value will be larger than 1 .

The $\chi^{2}$ diagram of the 1999 observation is influenced by strong aliases due to a regular observing scheme: the exposure time for each image was $1080 \mathrm{~s}$ and the elapsed time between two exposures about 300 to 400 s. Peaks in the $\chi^{2}$ diagram at about these periods, multiples of, differences between, and sums of them are most probably caused by the window function and not real variations in the light curves. Indeed the $\chi^{2}$ diagram of comparison star (A) shows peaks at multiples of $1400 \mathrm{~s}$ which are due to the starting of the exposures about every $1400 \mathrm{~s}$. They are also visible in the $\chi^{2}$ diagram of Grw $+70^{\circ} 8247$. 

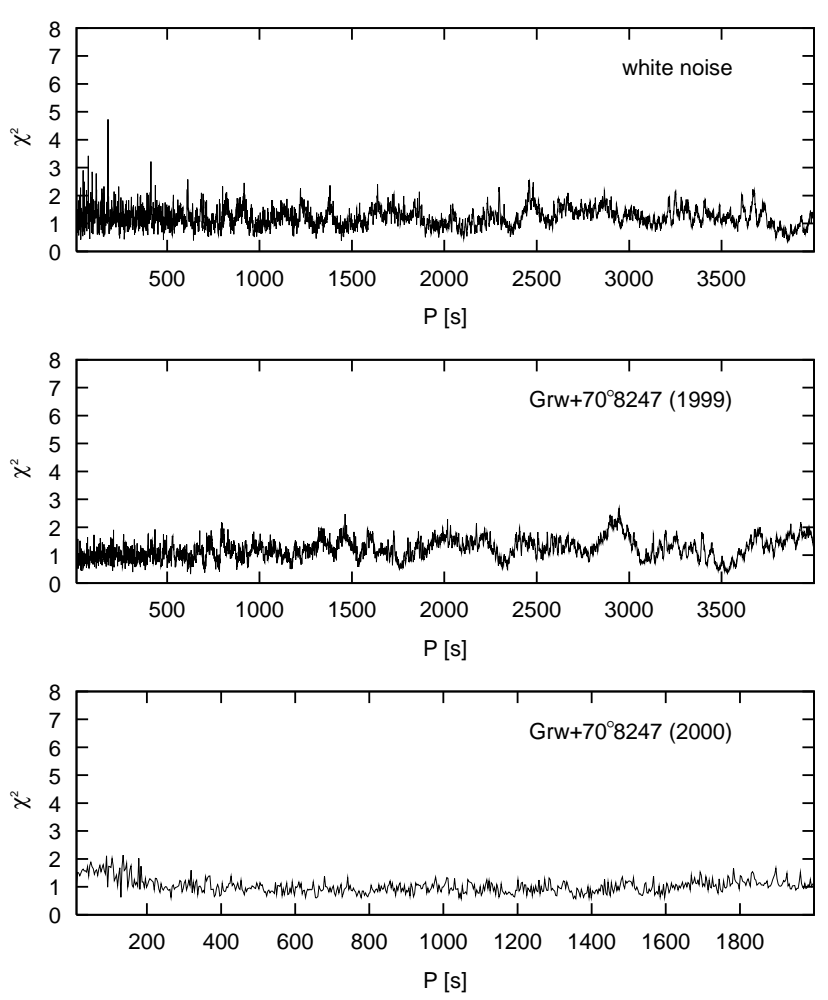

Fig. 3. $\chi^{2}$ diagram with trial periods up to $2000 \mathrm{~s}$ for Grw $+70^{\circ} 8247$ in 2000 and a retarder angle of 19 degrees (bottom), for the combined data of Grw $+70^{\circ} 8247$ in 1999 with trial periods up to $4000 \mathrm{~s}$ (middle), and for white noise with a mean polarization of $1 \%$ (top). Noise level and window function are identical to those of $\mathrm{Grw}+70^{\circ} 8247$ in 1999

No other, significant peaks could be found in the periodogram of Grw $+70^{\circ} 8247$ (Fig. 3 middle) pointing to any periodicity. For the 2000 observation we have minimized aliasing by variing the exposure times. As it can be seen in Fig. 3 (bottom) the corresponding $\chi^{2}$ diagram does not show any significant peaks.

In order to see which periods are detectable in a $\chi^{2}$ diagram we also constructed synthetic polarized data sets with the same noise level and the same window function as the observations assuming a sinusoidal variation of the linear polarization with different amplitudes of the polarization and periods between $10 \mathrm{~s}$ and $4000 \mathrm{~s}$ in steps of $1 \mathrm{~s}$ below $1800 \mathrm{~s}$ and in steps of $3 \mathrm{~s}$ above $1800 \mathrm{~s}$. Subsequently a $\chi^{2}$ test was performed for each period. For synthetic data constructed with the noise level and window function according to the 1999 observation periods could be detected in the $\chi^{2}$ diagram with an amplitude of about $30 \%-40 \%$ relative to the mean linear polarization of $1 \%$ (i.e. between $0.6 \%$ to $1.4 \%$ ). In most cases maxima in the $\chi^{2}$ diagram do not only appear at the period itself but also at multiples of the period. For trial periods larger than about $1000 \mathrm{~s}$ peaks in the $\chi^{2}$ diagram become broader, making the determination of the exact period difficult. Qualitatively the same results are obtained for the 2000 observation: periods with an amplitude of $50 \%$ of the mean linear polarization for an individual retarder setting can be detected and the
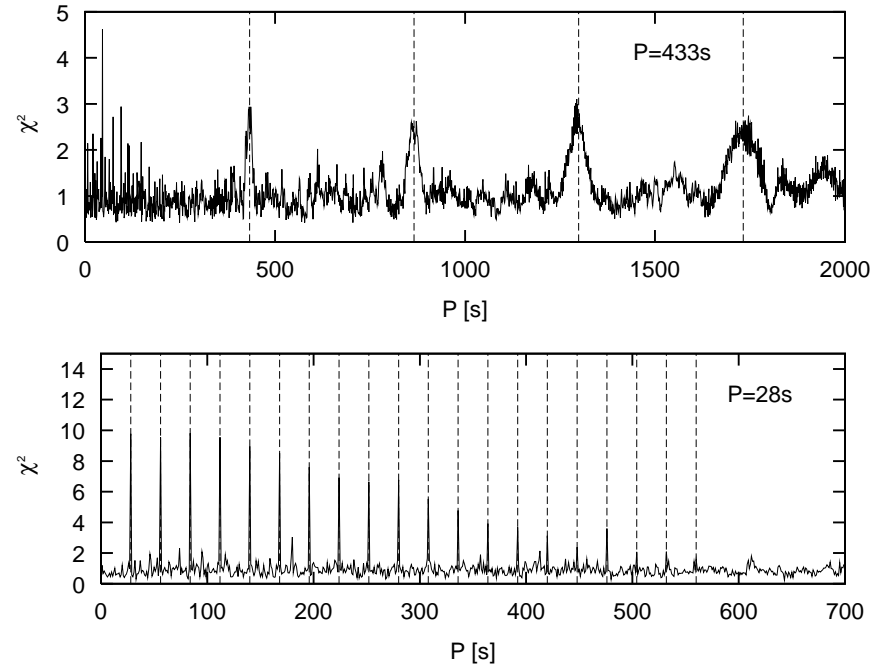

Fig. 4. $\chi^{2}$ diagrams for synthetic data of 1999 with a period of $28 \mathrm{~s}$ (bottom) and for synthetic data of 2000 with a period of $433 \mathrm{~s}$ (top). The variation was assumed to be sinusoidal with an amplitude of $30 \%$ (1999) and 50\% (2000) relative to the respective mean polarization. Noise level and window function were chosen according to the respective observations. Dashed lines mark multiples of the periods, which nicely coincide with the $\chi^{2}$ maxima

peaks become broader for periods above about $500 \mathrm{~s}$. As an example Fig. 4 shows the $\chi^{2}$ diagram of a synthetic data set for 1999 with a period of $28 \mathrm{~s}$ and of a synthetic data set (retarder angles of 19 and 199 degrees) for 2000 for a period of $433 \mathrm{~s}$. The linear polarization varies between $0.7 \%$ and $1.3 \%$ and between $0 \%$ and $1 \%$ for 1999 and 2000, respectively. Noise level and window function are identical to the respective observation. We therefore infer that we can detect periods with amplitudes of at least $40 \%$ of the mean linear polarization in the polarized light curves of Grw $+70^{\circ} 8247$. Since the $\chi^{2}$ diagram of the observed polarization of $\mathrm{Grw}+70^{\circ} 8247$ does not show any obvious peaks we conclude that no periodicities with amplitudes larger than $40 \%$ relative to the mean linear polarization are present. However, periodicities with smaller amplitudes or close to aliases cannot be excluded.

\section{Circular polarization data}

In a recent paper Berdyugin \& Piirola (1999) reported the detection of long-term variations in the polarization of GD 229. According to their Table 1 both the degree of linear polarization and the position angle increased in $B$, $V, I$, and $R$ for GD 229 between 1986 and 1996. The degree of circular polarization and its sign also changed in all band passes. We obtained circular polarization spectra of GD 229 and Grw $+70^{\circ} 8247$ in July 1989 at the Calar Alto $3.5 \mathrm{~m}$ telescope with a non-achromatic quarterwave plate implemented in the prime focus and in June 1998 with an achromatic quarter-wave plate implemented in the TWIN Cassegrain spectrograph (Graser 1998) at the same telescope. For a short description of the used 
Table 1. Circular polarization of GD 229 and Grw $+70^{\circ} 8247$

\begin{tabular}{lcccc}
\hline & \multicolumn{2}{c}{ GD 229 } & \multicolumn{2}{c}{ Grw $+70^{\circ} 8247$} \\
Time of observation & \multicolumn{2}{c}{ circular polarization } & \multicolumn{2}{c}{ circular polarization } \\
& $B$ & $V$ & $B$ & $V$ \\
\hline \hline $1986-1988^{1}$ & $-0.10 \pm 0.10$ & $+0.40 \pm 0.20$ & $-3.80 \pm 0.30$ & $-3.60 \pm 0.30$ \\
July 1989 & $-0.20 \pm 0.20$ & $+0.33 \pm 0.30$ & $-3.50 \pm 0.30$ & $-3.50 \pm 0.50$ \\
November $1996^{2}$ & $+0.69 \pm 0.04$ & $-0.37 \pm 0.05$ & - & - \\
June 1998 & $+1.00 \pm 0.20$ & $0.00 \pm 0.30$ & $-3.80 \pm 0.30$ & $-3.50 \pm 0.50$ \\
\hline
\end{tabular}

${ }^{1}$ West (1989); values for Grw $+70^{\circ} 8247$ are estimated from West's Fig. 1.

${ }^{2}$ Berdyugin \& Piirola (1999).

Zeeman analysers and data reduction procedures we refer to Friedrich et al. (1996) and Friedrich et al. (1997), respectively. In order to compare our data with other measurements of GD 229 (summarized in Berdyugin \& Piirola 1999 ) and Grw $+70^{\circ} 8247$ (summarized in West 1989) we folded the data with the band passes of Johnson $B$ and $V$ filters simulated by Gaussian profiles with respective FWHM. Circular polarization in the $V$ band of both observing runs is subject to large errors: the non-achromatic quarter-wave plate is already sensitive to linear polarization and the beam splitter of the TWIN spectrograph sits at $5500 \AA$. Thus the spectral range of the TWIN between $5400 \AA$ and $5600 \AA$ is of no use and was excluded for the calculation of the $V$ band. Nevertheless our results for GD 229 in the $V$ band show the same trend as the values in Berdyugin \& Piirola (1999) for respective times. They are given in Table 1 together with those from Berdyugin \& Piirola and West. From these data Berdyugin \& Piirola concluded that the rotation period for GD 229 could be about 100 years provided this variation is due to slow rotation.

However, the circular polarization of Grw $+70^{\circ} 8247$, also given in Table 1, does not show significant variations. From this result one can draw the conclusion that the rotation period of $\mathrm{Grw}+70^{\circ} 8247$ might be even longer than that of GD 229.

\section{Conclusion}

We could neither find short term variations with an amplitude larger than $40 \%$ relative to the mean linear polarization in our time resolved linear polarized light curves of Grw $+70^{\circ} 8247$ nor could we find variations in the circular polarization over a time span of 9 years. For GD 229, however, variations in the circular polarization were found, confirming the findings of Berdyugin \& Piirola (1999). On the basis of our current data we therefore conclude, that the rotation period of $\mathrm{Grw}+70^{\circ} 8247$ is much longer than that of GD 229. The measured mean linear polarization of $2.3 \% \pm 1 \%$ and mean polarization angle of 19 degrees \pm 5 degrees are within their errors consistent with older measurements of West (1989) which yielded $3.19 \% \pm 0.08 \%$ and 19.06 degrees \pm 0.76 degrees and can be considered as a point in favour of this conclusion.
However, according to new measurements of Berdyugin \& Piirola (2000) both the polarization and the polarization angle have marginally increased ( $3 \sigma$ and $5 \sigma$, respectively). Therefore the question whether Grw $+70^{\circ} 8247$ rotates slow or fast may still be open.

Acknowledgements. We want to thank the Calar Alto staff especially Francisco Prada for performing the observation in 1999. Work on magnetic white dwarfs in Kiel is supported by the DFG under KO-738/7-1. The observations at Calar Alto in 2000 were also supported by the DFG. We would like to thank the referee Dr. Berdyugin for valuable comments.

\section{References}

Angel, J. R. P., Borra, E. F., \& Landstreet, J. D. 1981, ApJS, 45,457

Bastien, P., Drissen, L., Menard, F., et al. 1988, AJ, 95, 900

Berdyugin, A. V., \& Piirola, V. 1999, A\&A, 352, 619

Berdyugin, A. V., \& Piirola, V. 2000, private communication

Bergeron, P., Fontaine, G., Brassard, P., et al. 1993, AJ, 106, 1987

Brecher, K., \& Chanmugam, G. 1978, ApJ, 221, 969

Burleigh, M. R., Jordan, S., \& Schweizer, W. 1999, ApJ, 510, L37

Friedrich, S., Östreicher, R., \& Schweizer, W. 1996, A\&A, 309, 227

Friedrich, S., König, M., \& Schweizer, W. 1997, A\&A, 326, 218

Graser, U. 1998, Cassegrain TWIN Spectrograph $3.5 \mathrm{~m}$ Telescope Calar Alto User's Manual, MPI-A Heidelberg

Jordan, S. 2000, in press

Kemp, J. C., Swedlund, J. B., Landstreet, J. D., \& Angel, J. R. P. 1970, ApJ, 161, L77

Koester, D., Dreizler, S., Weidemann, V., \& Allard, N. F. 1998, A\&A, 338, 612

Koester, D., \& Reimers, D. 1996, A\&A, 313, 810

Landstreet, J. D., \& Angel, J. R. P. 1975, ApJ, 196, 819

Meisenheimer, K. 1998, User Guide to the CAFOS 2.2, MPI-A Heidelberg

Meisenheimer, K. 2000, private communication

Minkowski, R. 1938, Ann. Rept. Dir. Mt. Wilson, 28

Pacini, F. 1970, Mem. Soc. Astron. Ital., 41, 445

Schmidt, G. D., \& Norsworthy, J. E. 1991, ApJ, 366, 270

Weidemann, V., \& Koester, D. 1983, A\&A, 121, 77

West, S. C. 1989, ApJ, 345, 511 